\title{
Variación espacio-temporal de Chironomidae (Diptera) bentónicos y derivantes en un arroyo serrano en Córdoba, Argentina
}

\author{
Juan P. Zanotto-Arpellino', Romina E. Principe ${ }^{1,2}$, Ana M. Oberto' \& Cristina M. Gualdoni
}

1. Universidad Nacional de Río Cuarto, Departamento de Ciencias Naturales, X5804, Río Cuarto, Córdoba, Argentina. (zanottojp@gmail.com)
2. Consejo Nacional de Investigaciones Científicas y Técnicas (CONICET).

\begin{abstract}
Spatio-temporal variation of benthic and drifting Chironomidae (Diptera) in a mountain stream in Córdoba, Argentina. Chironomidae is one of the most abundant and diverse families in freshwater ecosystems, however its highly complex systematic has led to its scarce development in many limnological studies. The aim of this study was to analyze benthic and drifting Chironomidae assemblages in a mountain stream of central Argentina assessing abundance, diversity and taxa composition in different fluvial habitats and seasons. Quantitative benthic and drift samples were taken in the Achiras stream (Córdoba, Argentina) in rifles and runs, and environmental variables were measured in each season between 2007 and 2008. A total of 25 taxa were registered belonging to four subfamilies. Benthic chironomids represented $19 \%$ of the macroinvertebrate community, whereas they constituted 33\% in drift. Thienemannimyia sp. dominated in benthos and Corynoneura sp. in drift. According to the two-way ANOVAs results, the highest taxonomic richness and benthic density were registered during the low flow period (autumn and winter) and the highest benthic diversity was found in riffles. On the contrary, in the drifting assemblage only evenness was different between habitats and among seasons. TWINSPAN analysis showed a spatio-temporal segregation of benthic samples, whereas drift samples were separated only temporally suggesting that the dislodgment of invertebrates downstream homogenize the spatial variation observed in benthos. Benthic and drift density varied in a similar manner through seasons and Jaccard coefficient showed a high similarity index between benthos and drift (86\%). This study allowed knowing the spatio-temporal dynamic of benthic and drifting Chironomidae in a mountain stream. The increase of knowledge in relation to taxonomy, biology and ecology of Chironomidae will allow to adequate and apply management and conservation strategies of lotic ecosystems in the central region of Argentina.
\end{abstract}

KEYWORDS. Fluvial habitats, lotic ecosystem, Tanypodinae, Orthocladiinae, Chironominae.

RESUMEN. Chironomidae es una de las familias más abundantes y diversas en los ecosistemas de agua dulce, sin embargo su complejidad sistemática ha sido motivo de su escaso desarrollo en muchos estudios limnológicos. El objetivo de este estudio fue analizar los ensambles de Chironomidae bentónicos y derivantes de un arroyo serrano de la región central de Argentina evaluando abundancia, diversidad y composición de especies en diferentes hábitats fluviales y distintas estaciones del año. Se tomaron muestras cuantitativas de bentos y deriva en el arroyo Achiras (Córdoba, Argentina) en hábitats de rabión y corredera, y se registraron variables ambientales en las cuatro estaciones del año, entre 2007 y 2008. Se registró un total de 25 taxones pertenecientes a cuatro subfamilias. Los quironómidos bentónicos constituyeron el 19\% de la comunidad de macroinvertebrados, mientras que en deriva representaron el 33\%. En el bentos dominó Thienemannimyia sp. y en la deriva, Corynoneura sp. De acuerdo con los resultados de ANOVAs de dos vías, los mayores valores de riqueza taxonómica y densidad bentónica se registraron en el periodo de aguas bajas (otoño e invierno) y la diversidad de quirónomidos bentónicos fue mayor en rabión. Por el contrario, para el ensamble de derivantes sólo la equitatividad fue diferente entre hábitats y entre estaciones. El análisis TWINSPAN mostró una separación espacio-temporal de las muestras de bentos, mientras que las muestras de deriva se segregaron sólo temporalmente lo que sugiere que el transporte aguas abajo de los invertebrados homogeniza la variación espacial observada en el bentos. La densidad de bentos y deriva varió de manera similar a través de las estaciones del año y el coeficiente de Jaccard presentó un alto índice de similitud entre bentos y deriva (86\%). Este trabajo posibilitó conocer la dinámica temporal y espacial de los quironómidos bentónicos y derivantes en un arroyo serrano. La ampliación del conocimiento taxonómico, biológico y ecológico de Chironomidae posibilitará adecuar e implementar estrategias de manejo y conservación de los ecosistemas lóticos en la región central de Argentina.

PALABRAS CLAVE. Hábitats fluviales, ecosistema lótico, Tanypodinae, Orthocladiinae, Chironominae.

Chironomidae constituye uno de los grupos del macrobentos de mayor interés para el completo conocimiento de los sistemas acuáticos continentales debido a su gran plasticidad ecológica y a su considerable abundancia y riqueza específica (PAGGI, 2009). Es el grupo de insectos más abundante en los sistemas acuáticos de agua dulce (CRANSTON, 1995), con fases larvarias que integran el bentos y se asocian a las macrófitas (Poi DE NeIFF \& NeIFF, 2006), constituyendo así un componente fundamental del ambiente fluvial (MarziaLi et al., 2006). Los estados inmaduros de Chironomidae tienen un rol destacado en las redes tróficas de las comunidades acuáticas (EPLER, 2001) y participan en las primeras etapas del proceso de degradación de la materia orgánica (PAGGI, 1998). Además, su rápido recambio generacional y su elevada tasa de crecimiento garantizan la disponibilidad de biomasa en el dinámico ecosistema acuático (MenzIE, 1981). Debido a su alta diversidad y abundancia y a los diferentes grados de tolerancia y sensibilidad a las distintas condiciones del medio, son considerados excelentes indicadores en estudios de calidad del agua, y en la tipificación del hábitat fluvial (PAGGi, 1999; Marziali et al., 2006).

La deriva de invertebrados es un aspecto muy importante a considerar en estudios ecológicos en sistemas fluviales (AlLAN \& CASTILLO, 2007) ya que está relacionada con la producción secundaria, además es fuente de alimento para peces y una manera efectiva de colonizar nuevas áreas (FLECKER, 1992; MiLNER, 1994). Numerosos estudios han analizado la deriva de invertebrados evaluando composición de especies y factores ambientales que la condicionan 
(Flecker, 1992; Koetsier \& Bryan, 1996; Gualdoni \& Corigliano, 1999; Barbero et al., 2013), sin embargo, pocos reportan información acerca del aporte de larvas de Chironomidae a la fracción derivante (Molineri, 2008; Marziali et al., 2009).

A pesar de la importancia del grupo y del considerable número de estudios que se han realizado en las últimas décadas en Limnología, existe aún escasa información sobre la biología, sistemática y ecología de estos insectos (Trivinho-Strixino \& Strixino, 1989). En América del Sur, el conocimiento de la diversidad de Chironomidae es aún escaso y se considera que la riqueza del grupo se encuentra por debajo del 50\% de los valores esperados (Ashe et al., 1987). En Argentina, el 22\% de las 170 especies hasta ahora descriptas no pueden reconocerse por ser sus descripciones insuficientes y por no contar con el material tipo. La causa principal de la frecuente omisión en la mayoría de los estudios limnológicos ha sido su complejidad sistemática, la cual está sometida a constantes y profundas revisiones.

Durante décadas en Argentina los estudios sobre Chironomidae fueron principalmente taxonómicos (PAGGI, 1998, 2009) aunque en los últimos años se han desarrollado estudios ecológicos (Medina \& PAGgi, 2004; Tejerina \& Molineri, 2007; Medina et al., 2008; PrinciPe et al., 2008). Sin embargo, poco se conoce respecto de los patrones de variación espaciotemporales de estos insectos en el bentos y su aporte a la deriva. En consecuencia, el objetivo de este estudio es analizar los ensambles de Chironomidae bentónicos y derivantes en un arroyo serrano de la región central de Argentina evaluando abundancia, diversidad y composición de especies en diferentes hábitats fluviales $\mathrm{y}$ distintas estaciones del año.

\section{MATERIALES Y MÉTODOS}

Área de estudio. El arroyo Achiras pertenece a la cuenca Achiras - del Gato (Córdoba, Argentina) (Fig. 1). Discurre al norte de la localidad de Achiras y en la zona de llanura, adopta la denominación de arroyo del Gato. Recorre unos $120 \mathrm{~km}$ y descarga en los Bañados del Tigre Muerto (Degiovanni, 2005). En el área de estudio el periodo lluvioso ocurre entre la primavera y el verano (Octubre-Marzo) con una precipitación anual de $725 \mathrm{~mm}$ (CABIDO et al., 2003).

El sitio de estudio está ubicado en la zona de ritron del arroyo Achiras a $810 \mathrm{msnm}$, y entre $33^{\circ} 09^{\prime} 24^{\prime \prime} \mathrm{S}$ y $64^{\circ} 59^{\prime} 05^{\prime}$ 'O (Fig. 1). En el tramo se distinguen dos tipos de hábitats: corredera y rabión. Las correderas son ambientes poco profundos donde predomina arena y grava, con moderada velocidad de corriente y turbulencia superficial escasa o nula. En estos ambientes los procesos de transporte y sedimentación superan a los de erosión. Mientras que los rabiones presentan fondo rocoso (bloque y roca), una velocidad de corriente elevada que producen considerable turbulencia con predominio de procesos erosivos.

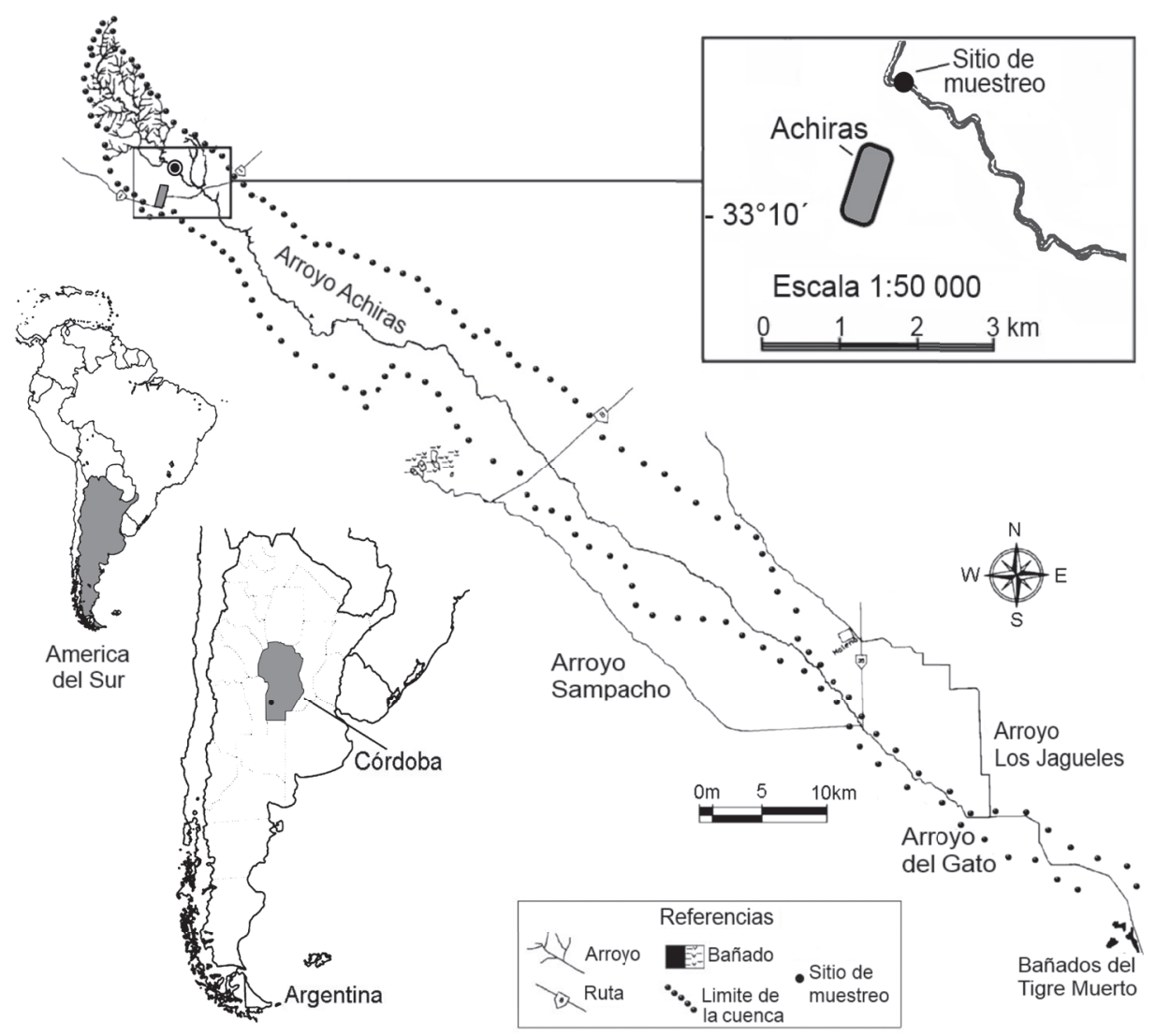

Fig. 1. Ubicación del sitio de muestreo en el arroyo Achiras (Córdoba, Argentina). 
El tramo de estudio es un ambiente natural con pocos signos de alteración. La vegetación está constituida por cinturones o pisos de vegetación que varían altitudinalmente (OGgero \& Arana, 2012). El sitio presenta una vegetación riparial continua, constituida por Geoffroea decorticans (Gillies ex Hook. \& Arn) Burkart., Celtis ehrembergiana (Klotzsch) Liebm, Cortaderia selloana (Schult. \& Schult. f.) Asch. \& Graebn, Vachellia caven (Molina) Seigler \& Ebinger var. dehiscens Burkart ex Ciald. y gramíneas, como Poa sp. Entre las especies introducidas se encuentra Salix sp.

Trabajo de campo y laboratorio. Se colectaron muestras en hábitat de corredera y rabión durante invierno (septiembre 2007), primavera (noviembre 2007), verano (marzo 2008) y otoño (mayo 2008). Para cada hábitat y estación del año se tomaron 3 muestras para el bentos y 3 para la fracción derivante. Se analizó un total de 24 muestras de bentos y 24 de deriva ( 3 muestras $\mathrm{x} 4$ estaciones x 2 hábitats).

El bentos se muestreó con redes de Surber $(300 \mu \mathrm{m}$, $0,09 \mathrm{~m}^{2}$ ) y la deriva con redes de $100 \mathrm{~cm}$ de largo, $300 \mu \mathrm{y}$ un área modificada de $24 \times 8 \mathrm{~cm}\left(0,019 \mathrm{~m}^{2}\right)$ (BARBERO et al., 2013). Se colocaron durante 30 minutos en la mitad de la columna de agua para excluir el neuston y el bentos, y entre las 9:00 y 11:00 hs para evitar la deriva comportamental. Las muestras se fijaron en campo con formol al $4 \%$.

Se registraron las variables hidráulicas del canal fluvial: profundidad, ancho, velocidad de corriente, y variables físicas y químicas: temperatura del agua, oxígeno disuelto, conductividad, $\mathrm{pH}$ y composición iónica. La profundidad y la velocidad de corriente se midieron empleando un velocímetro digital Global Flow Probe FP101-FP202, cada $50 \mathrm{~cm}$, en una transecta de ribera a ribera para caracterizar el tramo de estudio. Adicionalmente se registró la velocidad de corriente en la boca de cada red de deriva para el cálculo de la densidad de deriva. La temperatura del agua, el $\mathrm{pH}$ y la conductividad se midieron con sensores portátiles. Para la determinación de la composición química se colectó un litro de agua y se determinaron valores de carbonato, bicarbonato, sulfato, cloruro, sodio, potasio, calcio, magnesio, nitrato, nitrito, fluoruro y arsénico. La composición porcentual del sustrato de los hábitats se tomó de Gualdoni \& OBerto (2012).

En laboratorio se separaron las larvas de Chironomidae del resto de la muestra y se conservaron en alcohol etílico al 70\% para identificación y recuento. El material fue identificado hasta el máximo nivel taxonómico de determinación posible Wiederholm (1983), Epler (2001), y PAGGi (2009).

Análisis de datos. Se calcularon densidad bentónica y densidad de deriva, riqueza taxonómica, índice de diversidad de Shannon H' e índice de equitatividad J' (H'/Hmax). La densidad del bentos se calculó como el número de individuos por $\mathrm{m}^{2}$. La unidad que se utilizó para expresar cuantitativamente la deriva es la densidad de deriva, que es el número de invertebrados por unidad de volumen de agua filtrada (ElLiotT, 1970).
Para analizar las diferencias en riqueza, densidad, diversidad y equitatividad de bentos y deriva entre hábitats $y$ entre estaciones del año se realizaron ANOVAs de dos vías (factores: estación del año y hábitat). Se comprobaron los supuestos de homogeneidad de varianzas y normalidad y se transformaron a $\log _{10}$ la densidad bentónica y derivante para cumplir con estos supuestos. Se utilizó el test de Di Rienzo, Guzmán y Casanoves (DGC) para las comparaciones a posteriori. Para realizar ANOVAs se empleó el programa INFOSTAT Versión 2011 (Di RIENZO et al., 2010).

Para clasificar las muestras de quironómidos bentónicos y derivantes se utilizó TWINSPAN (Two way indicator species analysis) (HILL, 1979). Los taxones indicadores para cada grupo generado por TWINSPAN se calcularon utilizando el método IndVal (Indicator Value Method) propuesto por DufrêNe \& Legendre (1997). Se consideraron taxones indicadores a aquellos que presentaron valores indicadores (IV) significativos $(\mathrm{p}<0.05)$ mayores a 25 , se incluyen así los taxones presentes en más del $50 \%$ de las muestras de un grupo y con una abundancia relativa en ese grupo mayor al 50\% (DUFRÊNE \& LEGENDRE, 1997). El valor indicador IndVal para una especie dada, contrariamente a TWINSPAN, es independiente de otras abundancias relativas de especies y no hay necesidad de utilizar "pseudoespecies" (DOHET et al., 2002). El método IndVal y el análisis TWINSPAN se llevaron a cabo utilizando el programa PC-Ord para Windows 4.25 (MCCune \& MefFord, 1999).

Para comparar la similitud entre la comunidad bentónica y la fracción derivante se calculó el coeficiente de asociación de Jaccard, a partir de matrices de presencia/ ausencia (CRISCI \& López Armengol, 1983).

\section{RESULTADOS}

Variables hidráulicas y fisicoquímicas. El ancho húmedo máximo del canal fluvial fue $6,50 \mathrm{~m}$ en verano y el mínimo fue 4,45 m durante la primavera. Los valores medios de velocidad de corriente y profundidad que caracterizaron cada hábitat se resumen en la Tabla I. En el rabión donde predominan bloques $(50 \%)$ y rocas $(30 \%)$ se evidenció mayor turbulencia, mientras que en la corredera, caracterizada por sustrato de grava $(46 \%)$ y arena $(29 \%)$, presentó turbulencia escasa o nula.

Tab. I. Valores promedio ( \pm desvío estándar) de velocidad de corriente y profundidad para cada estación del año en los hábitats estudiados del arroyo Achiras (Córdoba, Argentina).

\begin{tabular}{lccc}
\hline & & Vel. $\left(\mathrm{m} \cdot \mathrm{seg}^{-1}\right)$ & Prof. $(\mathrm{cm})$ \\
\hline \multirow{4}{*}{ Corredera } & Otoño & $0,35( \pm 0,01)$ & $17,67( \pm 3,12)$ \\
& Invierno & $0,14( \pm 0,01)$ & $19,67( \pm 10,02)$ \\
& Primavera & $0,09( \pm 0,01)$ & $24,33( \pm 1,53)$ \\
& Verano & $0,18( \pm 0,04)$ & $38,33( \pm 5,13)$ \\
& Otoño & $0,18( \pm 0,02)$ & $47,50( \pm 3,12)$ \\
\multirow{4}{*}{ Rabión } & Invierno & $0,15( \pm 0,01)$ & $45,33( \pm 10,21)$ \\
& Primavera & $0,12( \pm 0,01)$ & $25,33( \pm 6,81)$ \\
& Verano & $0,34( \pm 0,06)$ & $39,67( \pm 6,81)$ \\
\hline
\end{tabular}


La temperatura del agua presentó el menor valor en otoño $\left(13^{\circ} \mathrm{C}\right)$ y el máximo $\left(20^{\circ} \mathrm{C}\right)$ en primavera. El tramo de estudio se caracterizó por un $\mathrm{pH}$ neutro ligeramente alcalino, aguas dulces, bicarbonatadas cálcicas, con

Tab. II. Valores promedio ( \pm desvío estándar) de las variables químicas del agua del arroyo Achiras, Córdoba, Argentina.

\begin{tabular}{lr}
$\mathrm{pH}$ & $7,78( \pm 0,79)$ \\
Conductividad a $20^{\circ} \mathrm{C}(\mu \mathrm{S} / \mathrm{cm})$ & $208,67( \pm 32,35)$ \\
Sólidos disueltos totales $(\mathrm{mg} / \mathrm{l})$ & $183,33( \pm 12,10)$ \\
Carbonato $(\mathrm{mg} / \mathrm{l})$ & $0,00( \pm 0,00)$ \\
Bicarbonato $(\mathrm{mg} / \mathrm{l})$ & $105,00( \pm 6,61)$ \\
Sulfato $(\mathrm{mg} / \mathrm{l})$ & $20,20( \pm 6,10)$ \\
Cloruro $(\mathrm{mg} / \mathrm{l})$ & $7,63( \pm 1,67)$ \\
Sodio $(\mathrm{mg} / \mathrm{l})$ & $12,73( \pm 3,69)$ \\
Potasio $(\mathrm{mg} / \mathrm{l})$ & $2,50( \pm 0,61)$ \\
Calcio $(\mathrm{mg} / \mathrm{l})$ & $27,73( \pm 5,21)$ \\
Magnesio $(\mathrm{mg} / \mathrm{l})$ & $5,37( \pm 1,45)$ \\
Nitrato $(\mathrm{mg} / \mathrm{l})$ & $1,63( \pm 1,18)$ \\
Nitrito $(\mathrm{mg} / \mathrm{l})$ & $0,00( \pm 0,00)$ \\
Fluoruro $(\mathrm{mg} / \mathrm{l})$ & $0,49( \pm 0,18)$ \\
Arsénico $(\mu \mathrm{g} / \mathrm{l})$ & $1,50( \pm 0,71)$ \\
Dureza Total $(\mathrm{meq} / \mathrm{l})$ & $1,83( \pm 0,32)$ \\
Alcalinidad TAC $(\mathrm{meq} / \mathrm{l})$ & $1,70( \pm 0,10)$ \\
\hline
\end{tabular}

mínimos tenores de F y As, y los compuestos nitrogenados fueron reducidos (Tab. II).

Comunidad bentónica. En el bentos se identificaron un total de 25 taxones de quironómidos, pertenecientes a cuatro subfamilias: Orthocladiinae (10 taxones), Tanypodinae (8 taxones), Chironominae (6 taxones) y Podonominae (1 taxón) (Tab. III). Los taxones más abundantes fueron: Tanytarsus sp., Thienemannimyia sp. y Corynoneura sp., y representaron el $67 \%$ de los quironómidos bentónicos. Para la subfamilia Podonominae, Podonomus sp. fue la única especie registrada y se colectó en invierno. En Chironominae, tanto Polypedilum sp. como Tanytarsus sp. presentaron las mayores densidades mientras que en Tanypodinae el taxón más abundante fue Thienemannimyia sp. En Orthocladiinae dominaron Corynoneura sp. y Cricotopus sp. 2.

Los ANOVAs de dos vías detectaron diferencias para densidad bentónica y riqueza taxonómica entre las estaciones del año (Tab. IV, Figs 2-7) mientras que la diversidad y equitatividad fueron diferentes entre hábitats y entre estaciones (Tab. IV, Figs 2-7). El análisis de

Tab. III. Lista sistemática de Chironomidae (Diptera) colectados en el arroyo Achiras (Córdoba, Argentina). Se presenta la suma de la densidad obtenida en las distintas estaciones del año en los distintos hábitats fluviales para bentos y deriva (* Según Ruiz-Moreno et al., 2000).

\begin{tabular}{|c|c|c|c|c|}
\hline & \multicolumn{2}{|c|}{ BENTOS } & \multicolumn{2}{|c|}{ DERIVA } \\
\hline & Corredera & Rabión & Corredera & Rabión \\
\hline \multicolumn{5}{|l|}{ PODONOMINAE } \\
\hline Podonomus sp. & 0,00 & 11,11 & 0,68 & 0,00 \\
\hline \multicolumn{5}{|l|}{ CHIRONOMINAE } \\
\hline Chironominae género $A^{*}$ & 0,00 & 0,00 & 0,00 & 0,57 \\
\hline Chironomus sp. & 3,70 & 22,22 & 0,00 & 0,00 \\
\hline Polypedilum sp. & 103,70 & 2985,19 & 8,82 & 9,45 \\
\hline Dicrotendipes sp. & 11,11 & 14,81 & 0,92 & 0,57 \\
\hline Tanytarsus sp. & 11140,74 & 2529,63 & 20,61 & 27,47 \\
\hline Rheotanytarsus sp. & 381,48 & 1070,37 & 21,14 & 18,33 \\
\hline Pseudochironomus sp. & 0,00 & 33,33 & 0,68 & 0,00 \\
\hline \multicolumn{5}{|l|}{ TANYPODINAE } \\
\hline Tanypodinae indet 1. & 40,74 & 3,70 & 2,05 & 0,00 \\
\hline Tanypodinae indet 2. & 0,00 & 7,41 & 0,00 & 0,00 \\
\hline Ablabesmyia sp. & 74,07 & 55,56 & 0,00 & 0,63 \\
\hline Labrundinia sp. & 114,81 & 22,22 & 1,11 & 0,00 \\
\hline Larsia sp. & 2040,74 & 270,37 & 0,68 & 5,04 \\
\hline Pentaneura sp. & 7,41 & 14,81 & 0,68 & 0,00 \\
\hline Thienemannimyia sp. & 6107,41 & 1018,52 & 14,54 & 11,98 \\
\hline Djalmabatista sp. & 92,59 & 14,81 & 0,00 & 0,72 \\
\hline \multicolumn{5}{|l|}{ ORTHOCLADINAE } \\
\hline Corynoneura sp. & 488,89 & 5174,07 & 38,93 & 91,54 \\
\hline Onconeura sp. & 107,41 & 1477,78 & 15,23 & 24,72 \\
\hline Thienemanniella sp. & 59,26 & 448,15 & 11,03 & 15,81 \\
\hline Lopescladius sp. & 337,04 & 14,81 & 0,00 & 0,00 \\
\hline Cricotopus indet. & 0,00 & 0,00 & 5,09 & 3,63 \\
\hline Cricotopus sp. 1 & 11,11 & 37,04 & 3,92 & 1,78 \\
\hline Cricotopus sp. 2 & 351,85 & 840,74 & 18,82 & 47,95 \\
\hline Cricotopus sp. 3 & 85,19 & 111,11 & 6,73 & 1,14 \\
\hline Orthocladius sp. & 14,81 & 3,70 & 3,30 & 0,95 \\
\hline Parametriocnemus sp. 1 & 33,33 & 1244,44 & 1,70 & 3,03 \\
\hline Parametriocnemus sp. 2 & 7,41 & 266,67 & 2,05 & 0,00 \\
\hline CHIRONOMIDAE (pupas) & 85,19 & 129,63 & 9,63 & 26,81 \\
\hline
\end{tabular}



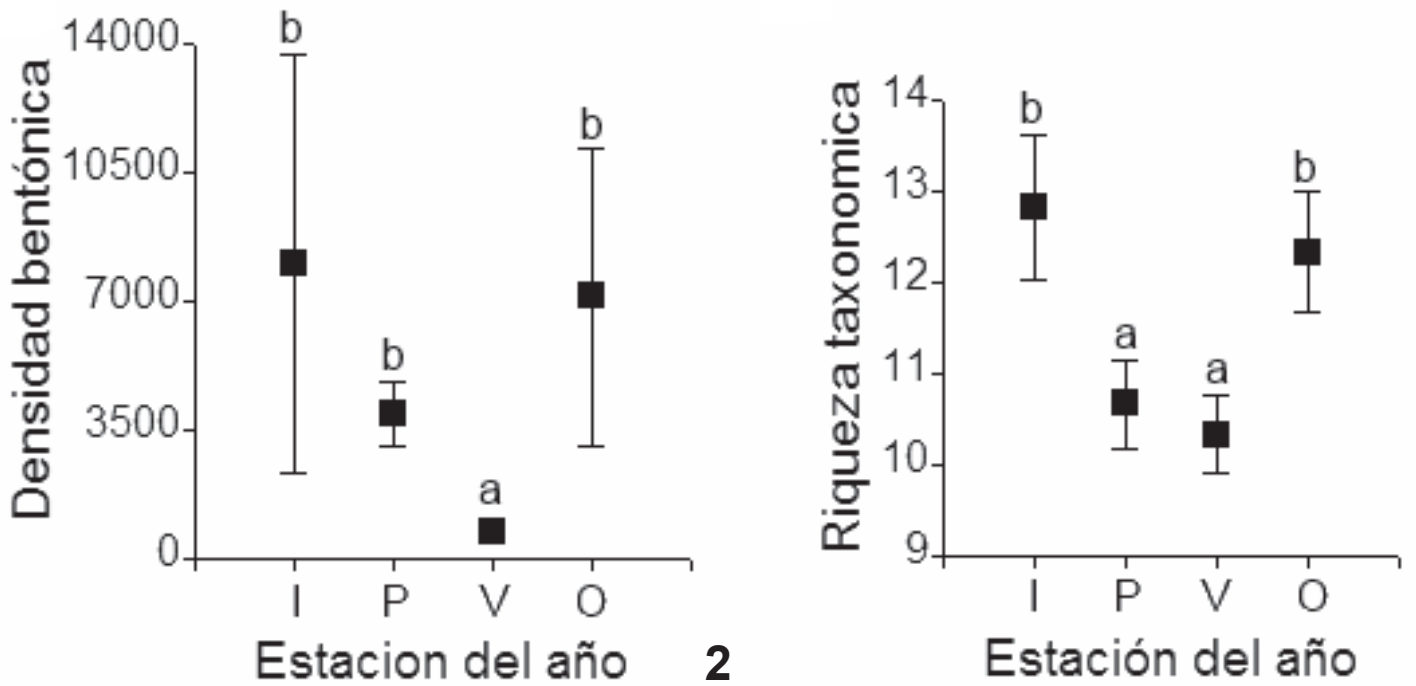

2
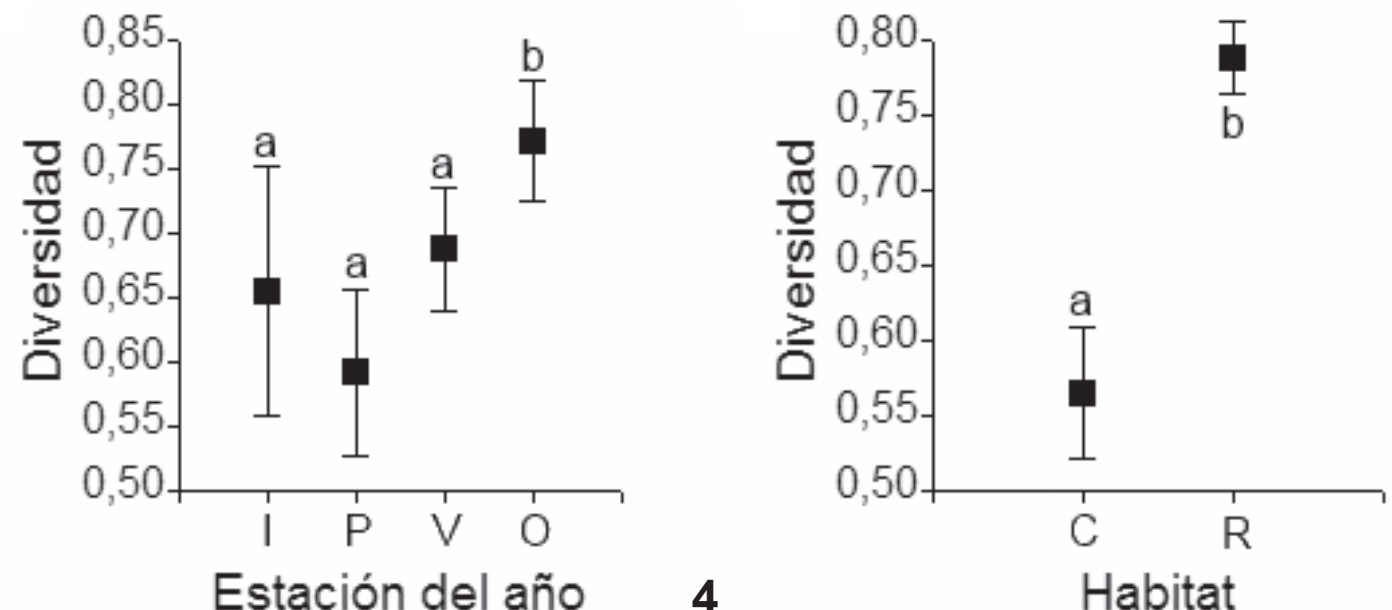

4

Habitat

5

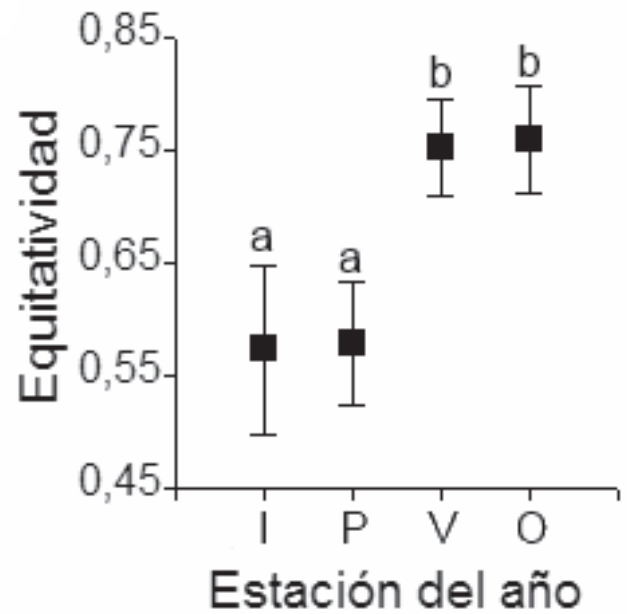

6

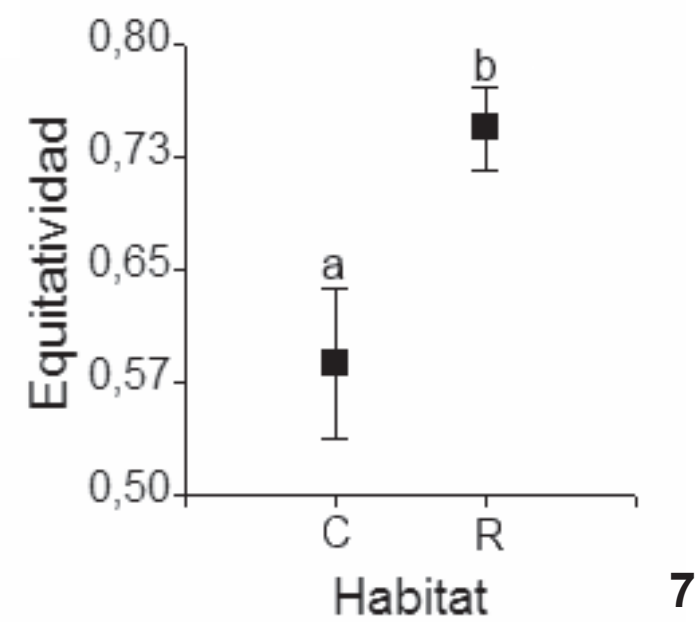

Figs 2-7. Valores medios y error estándar de las variables biológicas bentónicas que presentaron diferencias significativas con los ANOVAs. Se presenta la variación estacional de densidad bentónica (2); riqueza taxonómica (3); diversidad (4) y equitatividad (6); y la variación entre los hábitat de corredera y rabión de diversidad (5) y equitatividad (7). Letras diferentes indican diferencias significativas de acuerdo con los test a posteriori (DGC $\mathrm{p}<0,05)$. 
TWINSPAN aplicado a las muestras de bentos separó, en la primera división jerárquica, las muestras de verano y otoño de las muestras colectadas en invierno y primavera (Fig. 8). En invierno y primavera los taxones indicadores fueron Tanytarsus sp., Rheotanytarsus sp., Djalmabatista sp., Ablabesmyia sp. y Lopescladius sp.; mientras que para verano y otoño los indicadores fueron Thienemanniella sp., Onconeura sp. y Cricotopus sp. 2. La siguiente división de TWINSPAN separó los hábitats de corredera y rabión. Las especies indicadoras de rabión en invierno y primavera fueron Thienemannimyia sp. y Lopescladius sp. y las de corredera fueron Polypedilum sp., Chironomus sp. y Pseudochironomus sp. En verano y otoño sólo se encontraron especies indicadoras en rabión (Fig. 8).
Fracción derivante. En el tramo estudiado del arroyo Achiras, la mayoría de los taxa hallados en el bentos estuvieron representados en la deriva. Se identificaron un total de 24 taxones, 10 de Orthocladiinae, 7 de Tanypodinae, 6 de Chironominae y 1 de Podonominae (Tab. III). Los taxones más abundantes fueron: Corynoneura sp., Cricotopus sp. 2, Tanytarsus sp., Onconeura sp. y Rheotanytarsus $\mathrm{sp}$. A excepción de los dos primeros, las densidades de deriva de estos taxones no superaron los 23 ind. $/ \mathrm{m}^{3}$. En cambio, Cricotopus sp. 2 y Corynoneura sp. representaron $45,87 \%$ de quironómidos derivantes, destacándose elevados registros en rabión durante el otoño.

En Podonominae sólo se registró Podonomus sp., mientras que en la subfamilia Chironominae las

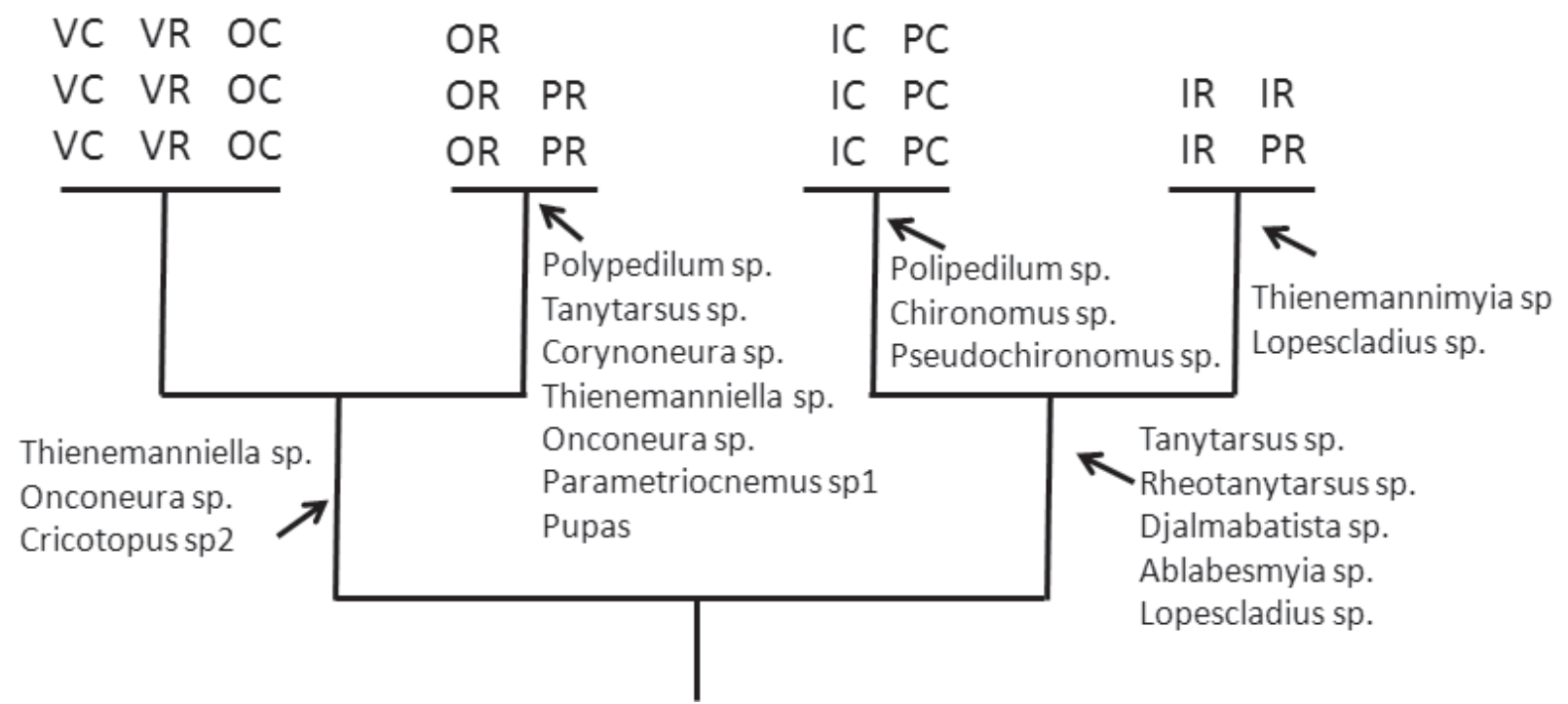

Fig. 8. Dendrograma resultante de la aplicación del TWINSPAN a las muestras de quironómidos bentónicos. Los taxones indicadores que se presentan fueron obtenidos por el método IndVal (IV > 75 y p < 0,05). Referencia: V: verano, O: otoño, I: invierno, P: primavera, C: corredera, R: rabión.

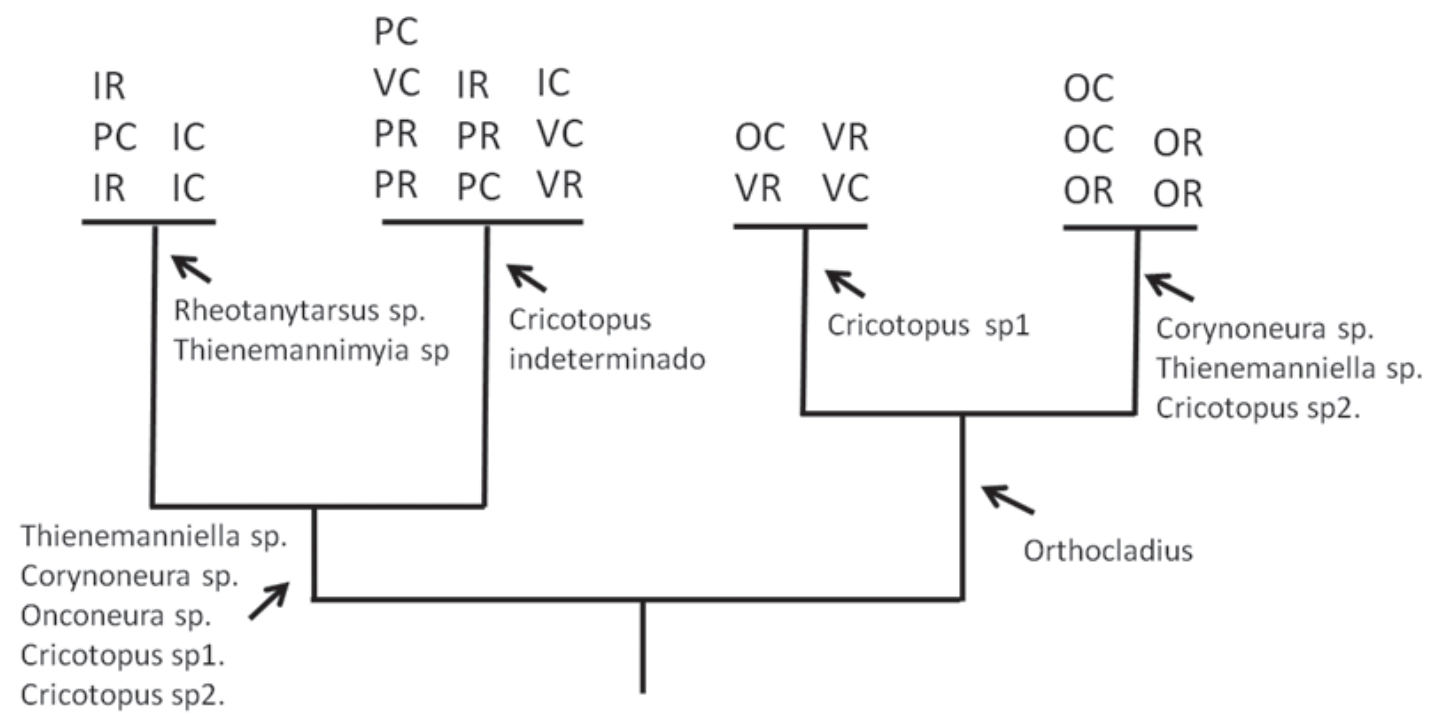

Fig. 9. Dendrograma resultante de la aplicación del TWINSPAN a las muestras de quironómidos derivantes. Los taxones indicadores que se presentan fueron obtenidos por el método IndVal (IV $>75$ y p < 0,05). Referencia: V: verano, O: otoño, I: invierno, P: primavera, C: corredera, R: rabión. 
mayores densidades correspondieron a Rheotanytarsus sp. y Tanytarsus sp. en corredera durante el invierno. Entre los Tanypodinae, la especie más abundante fue Thienemannimyia sp. en invierno. También se hallaron especies raras que ocurrieron sólo en una estación y un hábitat tales como Djalmabatista sp., Ablabesmyia sp. Labrundinia sp., Larsia sp., y Pentaneura sp. (Tab. III). Entre los Orthocladiinae, Cricotopus sp. 2 y Corynoneura sp. presentaron su mayor abundancia en otoño y las pupas pertenecientes a todas las subfamilias fueron abundantes en primavera y verano.

Los ANOVAs no detectaron diferencias significativas en densidad de deriva, ni en riqueza taxonómica, ni en diversidad. Sólo se registraron efectos significativos para hábitat y estación del año sobre la equitatividad (Tab. IV). Las correderas fueron los hábitats más equitativos con un valor medio de 0,88 mientras que en rabión la media fue 0,80 (test a posteriori DGC, $\mathrm{p}<0,05$ ). Al comparar las estaciones del año se registró el valor mínimo en el otoño $(0,70)$ y los máximos en el resto de las estaciones (invierno: 0,85; primavera: 0,93; verano: 0,88 ) (test $a$ posteriori $\mathrm{DGC}, \mathrm{p}<0,05)$. El análisis TWINSPAN mostró que las muestras de deriva se agruparon principalmente en función de las distintas estaciones del año aunque las separaciones no fueron tan evidentes como en el bentos (Fig. 9). De manera similar a los resultados obtenidos con las muestras de bentos, se observó una tendencia de agrupamiento de las muestras de verano y otoño, las cuales presentaron un solo taxón indicador (Orthocladius sp.). El grupo de muestras de invierno y primavera presentó a su vez 5 taxones indicadores (Fig. 9) y contrariamente a lo registrado para las muestras de bentos, las de deriva no se segregaron en función de los hábitats fluviales.

Bentos y deriva. La variación de las densidades de bentos y deriva a través de las distintas estaciones del año presentó un patrón similar (Fig. 10), especialmente en el hábitat de rabión. El coeficiente de Jaccard aplicado para comparar ambas fracciones proporcionó un índice de similitud del 86\%. Los quironómidos del bentos constituyeron el $19 \%$ de la comunidad de macroinvertebrados, mientras que en deriva representaron el $33 \%$.

En el bentos, Chironominae dominó en invierno, Tanypodinae en primavera, mientras que Orthocladiinae estuvo altamente representado en verano y otoño (Fig. 11). En la deriva, la subfamilia Chironominae representó más del $50 \%$ de los quironómidos en invierno, el cual se fue reduciendo a través de las estaciones y fue reemplazada por Orthocladiinae, que dominó la deriva de primavera, verano y otoño. Tanypodinae presentó una baja abundancia proporcional en deriva (Fig. 12). Al considerar la distribución de las subfamilias en los distintos hábitats se observó que en el bentos, las correderas estuvieron dominadas por Chironominae y Tanypodinae, en cambio, en rabión el mayor porcentaje correspondió a Orthocladiinae (Fig. 13). En la deriva, Orthocladiinae fue dominante en ambos hábitats (Fig. 14).

\section{DISCUSIÓN}

Comunidad bentónica. En el sitio estudiado se han registrado los mismos taxones citados en otros sistemas lóticos de la región central de Argentina (PrinciPe et al., 2007, 2008; BARBERO et al., 2013). La densidad relativa total estuvo dominada por los Chironominae (47\%), continuando con los Orthocladiinae (28\%) y Tanypodinae (25\%). Algunos estudios en cursos de agua de Argentina también reportaron una dominancia de Chironominae $(80 \%)$ y una baja abundancia relativa de Orthocladiinae (8,2\%) (Medina \& Paggi, 2004; Medina et al., 2008). De acuerdo con AsHe et al., (1987), la proporción de subfamilias de Chironomidae encontrada en el arroyo Achiras presenta similitudes con la descripta para la zona tropical-subtropical, y corresponde a una fauna de taxones euritérmicos cálidos, con Chironominae dominante y un porcentaje menor compuesto por taxones estenotérmicos

Tab. IV. Resultados de ANOVAs de dos vías sobre los atributos estructurales de la comunidad bentónica y de la fracción derivante. Se presentan en negrita los valores de $\mathrm{p}$ significativos $(\mathrm{p} \leq 0,05)$.

\begin{tabular}{|c|c|c|c|c|c|c|}
\hline \multirow{2}{*}{ Fuente de variación } & \multicolumn{3}{|c|}{ Bentos } & \multicolumn{3}{|c|}{ Deriva } \\
\hline & G. L. & $\mathrm{F}$ & $\mathrm{p}$ & G. L. & $\mathrm{F}$ & $\mathrm{p}$ \\
\hline \multicolumn{7}{|l|}{ Densidad } \\
\hline Hábitat & 1 & 0,12 & 0,7386 & 1 & 3,14 & 0,0953 \\
\hline Estación & 3 & 4,56 & 0,0172 & 3 & 2,40 & 0,1057 \\
\hline Hábitat x Estación & 3 & 1,54 & 0,2433 & 3 & 1,50 & 0,2526 \\
\hline \multicolumn{7}{|l|}{ Riqueza taxonómica } \\
\hline Hábitat & 1 & 2,47 & 0,1356 & 1 & 0,26 & 0,6194 \\
\hline Estación & 3 & 4,43 & 0,0190 & 3 & 1,68 & 0,2111 \\
\hline Hábitat x Estación & 3 & 1,16 & 0,3545 & 3 & 0,88 & 0,4699 \\
\hline \multicolumn{7}{|l|}{ Diversidad } \\
\hline Hábitat & 1 & 31,72 & $<0,0001$ & 1 & 0,24 & 0,6329 \\
\hline Estación & 3 & 3,65 & 0,0354 & 3 & 1,4 & 0,2788 \\
\hline Hábitat x Estación & 3 & 2,91 & 0,0664 & 3 & 1,97 & 0,1586 \\
\hline \multicolumn{7}{|l|}{ Equitatividad } \\
\hline Hábitat & 1 & 12,89 & 0,0024 & 1 & 11,81 & 0,0034 \\
\hline Estación & 3 & 5,80 & 0,0070 & 3 & 17,68 & $<0,0001$ \\
\hline Hábitat x Estación & 3 & 1,57 & 0,2360 & 3 & 3,15 & 0,0539 \\
\hline
\end{tabular}



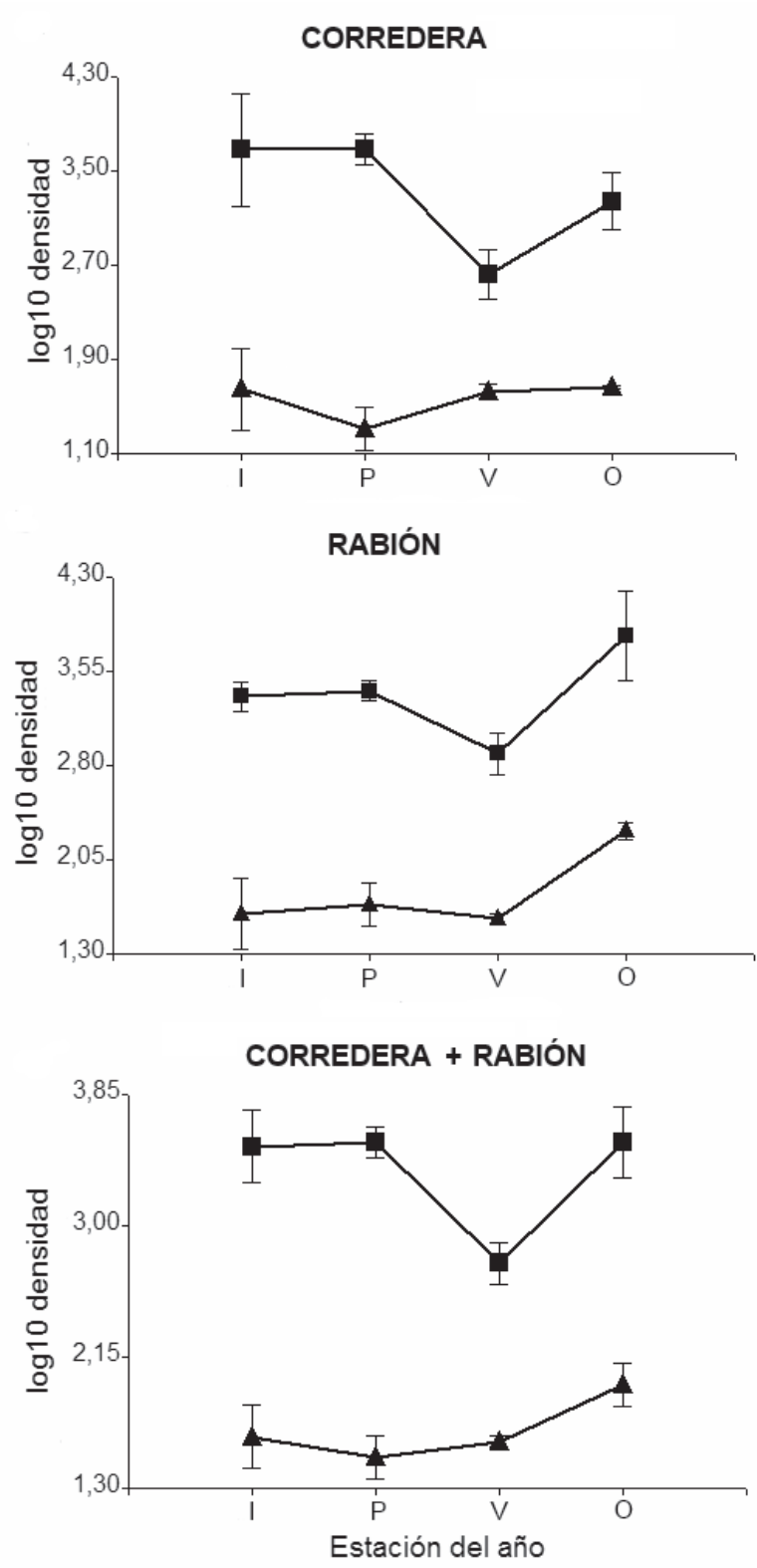

Fig. 10. $\log _{10}$ de la abundancia de quironómidos bentónicos y derivantes en los hábitats en estudio para cada estación del año. A: Corredera; B: Rabión y C: Sumatoria de abundancia en ambos hábitats Referencia: V: verano, $\mathrm{O}$ : otoño, I: invierno, $\mathrm{P}$ : primavera

fríos (Podonominae y Orthocladiinae) (CRANSTON, 1995). Los taxones más abundantes del arroyo Achiras fueron Tanytarsus sp., Corynoneura sp. y Thienemannimyia $\mathrm{sp}$., en coincidencia con lo registrado en arroyos de las Yungas por Tejerina \& Molineri (2007). Medina \& PagGi (2004) reportan también a Tanytarsus sp. como dominante mientras que en otros estudios de la provincia de Córdoba dominaron distintos géneros: Polypedilum (Príncipe et al., 2008; Gualdoni et al., 2009), Onconeura y Rheotanytarsus (BARBero et al., 2013); Rheotanytarsus (GUALDONI \& OвеRTo, 2012). Tanytarsus es un género euritópico que se encuentra en diferentes hábitats, incluyendo aguas salobres (Pinder \& Reiss, 1983; Epler, 2001). En el tramo estudiado, Tanytarsus estuvo presente en corredera y rabión y en casi todas las estaciones. En otros arroyos serranos, PRINCIPE et al. (2007) registraron este género principalmente asociado a correderas. Las larvas de Corynoneura se pueden encontrar en casi todos los tipos de hábitats, desde agua estancada hasta arroyos de montaña con alta velocidad de corriente (Epler, 2001). En el arroyo Achiras, Corynoneura sp. estuvo presente en todas las estaciones del año y en otros arroyos serranos se registró en hábitats de sustrato grueso y flujo suave (PrinciPe et al., 2007). En el arroyo estudiado, Thienemannimyia sp. predominó en corredera durante la primavera y en rabión en el otoño mientras que en otros estudios se ha registrado en hábitats de sedimento fino (Principe et al., 2007; Trivinho- Strixino, 2011).

El análisis de las variables estructurales del ensamble de quironómidos del arroyo Achiras indicó que los mayores valores de riqueza taxonómica y densidad bentónica se registraron en el periodo de aguas bajas (otoño e invierno). El arroyo posee un régimen hídrico freáticopluvial, con caudales menores y más estables durante este periodo, cuando las lluvias son escasas y el arroyo depende principalmente del aporte freático (GUALDONI \& OвеRTO, 2012). En estos periodos hay más posibilidades de colonización y son esperables aumentos en el número de taxones y de sus densidades (PofF \& ZimMERMAn, 2010).

Cuando se compararon los hábitats, se encontraron diferencias significativas sólo para la diversidad que fue mayor en rabión. Los rabiones son unidades geomorfológicas más complejas que ofrecen numerosos nichos para la macrofauna bentónica, actúan como refugios ante inundaciones y depredadores, y exhiben condiciones adecuadas para la alimentación. Por otra parte, los hábitats con sustrato de granulometría fina, como correderas, resultan inestables y la disminución de la disponibilidad de materia orgánica conduce a valores bajos de riqueza y diversidad (HAwKINS, 1984).

El análisis TWINSPAN separó en el primer nivel jerárquico las muestras de verano y otoño de las colectadas en invierno y primavera, mientras que en el segundo nivel, se separaron en función de los distintos hábitats. La granulometría del sustrato y la velocidad de la corriente son factores que afectan la distribución de los quironómidos (Lencioni \& Rossaro, 2005; Principe et al., 2008) y pueden actuar conjuntamente para determinar sus preferencias de hábitat (Sanseverino \& Nessimian, 1998). Asimismo, las variaciones estacionales han sido atribuidas al voltinismo $\mathrm{y}$ al efecto de la temperatura sobre ciclos de vida de las poblaciones (CORTES, 1992).

Fracción derivante. En la bibliografía se aprecia una gran variabilidad en los valores de densidad de deriva, no sólo entre ríos, sino también a lo largo del año (Gualdoni \& Corigliano, 1999). Se ha estimado que las densidades medias de deriva de macroinvertebrados varían entre 100 y $1000 \mathrm{ind} / \mathrm{m}^{3}$ (Allan, 1987). Los valores de Chironomidae en el tramo estudiado del arroyo Achiras son moderadamente elevados, si se tiene en cuenta que la densidad media fue de $508 \mathrm{ind} / \mathrm{m}^{3}$ y corresponde a una única familia dentro de la comunidad de macroinvertebrados. Las variaciones pueden estar condicionadas por la temperatura, 


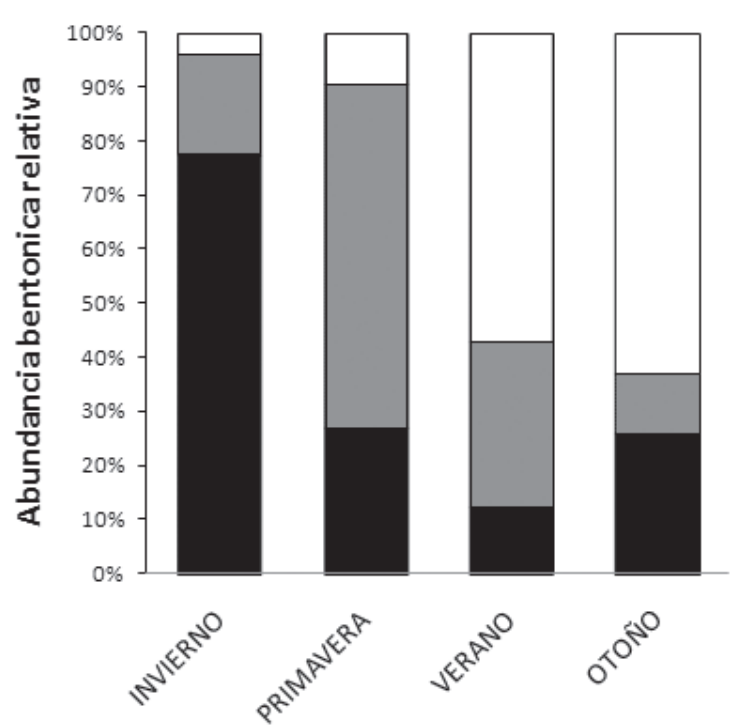

Estacion del año

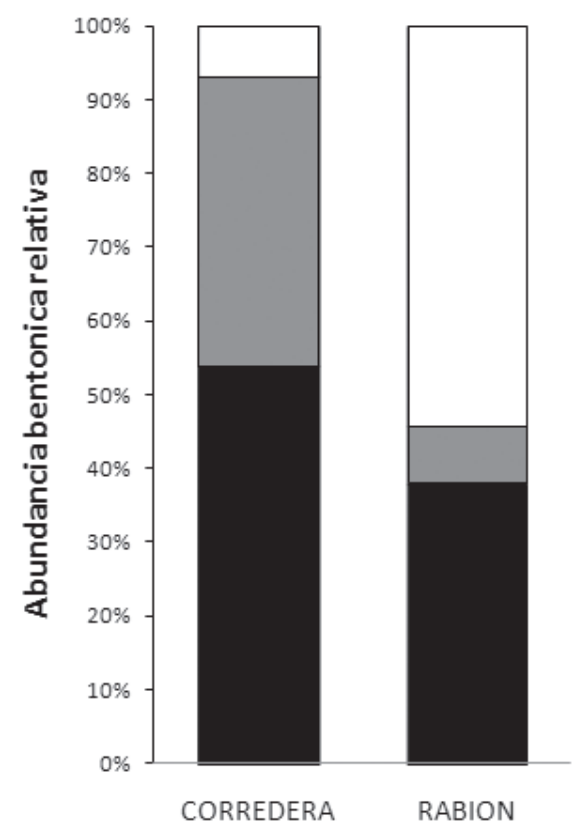

Habitats
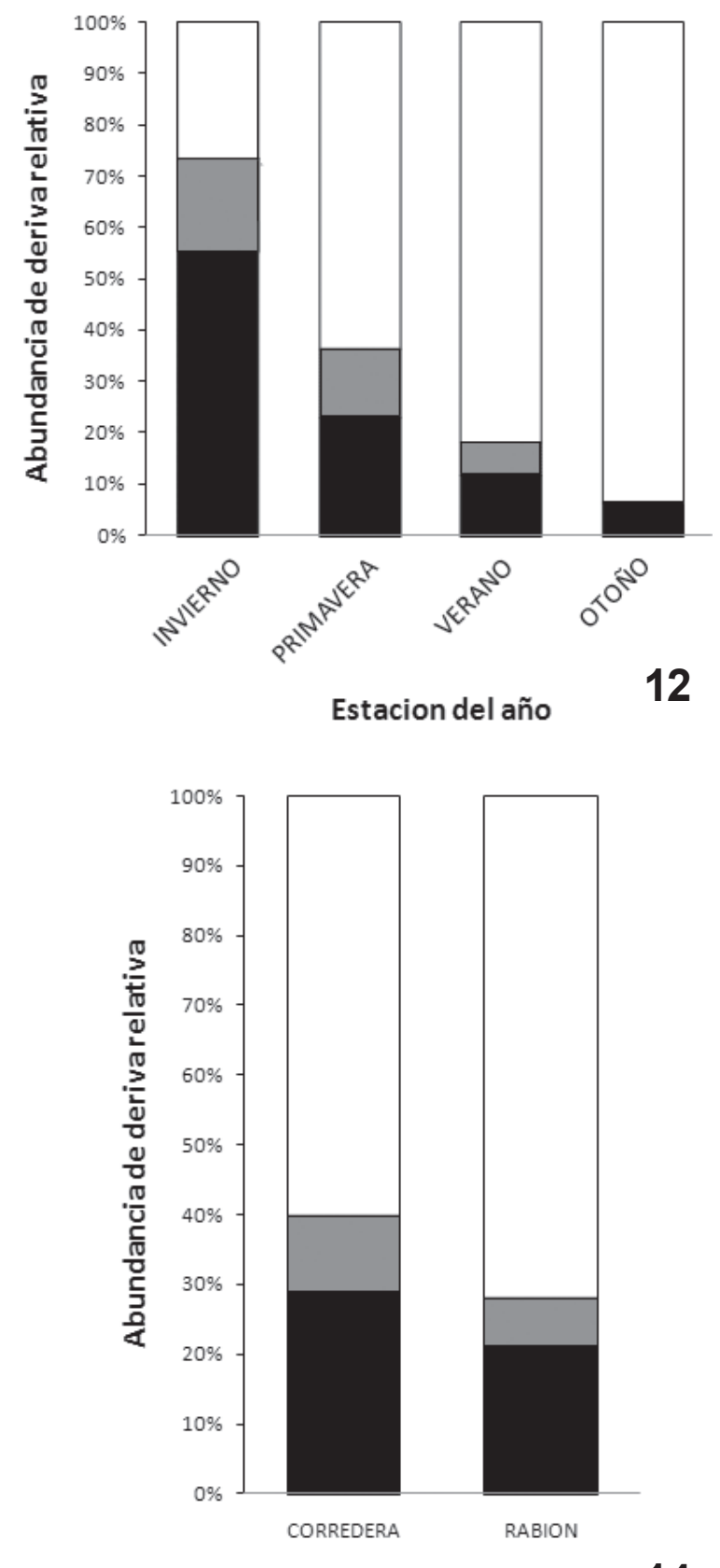

Habitats

\section{$\square$ Orthocladinae $\square$ Tanypodinae $\quad$ Chironominae}

Figs 11-14. Abundancia relativa bentónica y derivante de las subfamilias de quironómidos del arroyo Achiras, según las estaciones del año y según los distintos hábitats considerados.

la velocidad de corriente, el sustrato y el ciclo de vida. Los valores reportados en estudios de deriva son muy variables evidenciando la multiplicidad de factores que pueden afectar a este proceso.

Las especies más abundantes en la deriva del arroyo Achiras fueron Corynoneura sp. y Cricotopus sp. 2. Datos similares fueron registrados por Principe \& Corigliano (2006) y BARBERO et al. (2013). Corynoneura y Cricotopus son géneros con muchas especies, cosmopolitas y las larvas son comunes en los ambientes lóticos (EPLER, 2001) además, son muy propensas a ser arrastradas río abajo por la corriente (C. M. Gualdoni, obs. pers.).

El desprendimiento de los organismos del sustrato y su consiguiente arrastre río abajo puede deberse a diversos motivos (Waters, 1965). Sin embargo, en este trabajo la posibilidad de deriva catastrófica es excluida ya que los muestreos en periodo de aguas altas fueron realizados siete días después del evento de máximo caudal, tiempo 
necesario para que las densidades poblacionales se normalicen (GHetTI, 1986). Por otra parte, el horario en que se realizaron las colectas también permite descartar la posibilidad de deriva comportamental. Numerosos factores morfológicos y etológicos influyen en el desprendimiento de los organismos y su posterior arrastre aguas abajo (RADER, 1997). La predisposición a la deriva está en relación con la forma corporal, con las adaptaciones morfo-etológicas, con el microhábitat preferencial de cada especie y la velocidad de corriente óptima dentro de cada unidad de hábitat (RADER, 1997). Debido a la escasa capacidad de natación que poseen los quironómidos, han sido clasificados como no nadadores (RADER, 1997). Probablemente, los movimientos ondulatorios con poca direccionalidad y la baja eficiencia de retorno, mantuvieron a estos organismos en deriva por más tiempo y originó las relativamente altas densidades registradas.

El análisis TWINSPAN mostró que el ensamble de quironómidos derivantes varió principalmente en función de las distintas estaciones del año. La deriva de macroinvertebrados presenta un patrón anual debido a la variación en el ciclo de vida de las especies y de la densidad bentónica; y a los cambios estacionales en la temperatura del agua y velocidad de corriente (HILDEBRAND, 1974). La historia de vida de los organismos bentónicos influye en la microdistribución de los efectivos por clases de edad y es uno de los factores que produce las variaciones estacionales en deriva, que fluctúa con cada estadio del ciclo biológico (LEHMKUHL \& ANDERSON, 1972). El riesgo a derivar se eleva en las etapas de pre-emergencia, cuando los individuos con formas adultas aéreas, abandonan la protección del fondo para alcanzar la superficie del agua. En el arroyo estudiado se registró un bajo número de larvas derivantes en primavera y verano, mientras que el estado de pupa fue más abundante, probablemente debido a que es el periodo de emergencia del imago y a que las pupas presentan un gran riesgo de ser transportadas por la corriente durante el ascenso hacia la superficie. Por otra parte, el análisis TWINSPAN no mostró agrupamientos de las muestras de deriva en función de los distintos hábitats fluviales. Resultados similares fueron reportados por BARBERO et al. (2013) y sugieren que el transporte aguas abajo de los invertebrados homogeniza la variación espacial observada en el bentos.

Relación Bentos-deriva. En el arroyo Achiras, la familia Chironomidae aportó significativamente a la densidad total de la comunidad, contribuyendo con el 19\% de los organismos bentónicos y el 33\% de los derivantes. Otros estudios registran a los quironómidos como la familia más abundante y pueden representar el $50 \%$ a $78 \%$ de los invertebrados bentónicos (LENCIONI \& RoSSARO, 2005; GUALDONI et al., 2009) y del $14 \%$ al $85 \%$ de los invertebrados en la deriva (Corigliano et al., 1987; MARZIALI et al., 2009).

La importancia de la densidad bentónica, como uno de los principales factores que afecta la densidad de deriva, ha sido investigada en diferentes ambientes y las conclusiones no siempre fueron concordantes. Algunos autores hallaron una relación entre ambas variables (Allan, 1987), otros consideran que las dos fracciones presentan diferentes patrones de distribución y abundancia (KoETSIER \& Bryan, 1996). En nuestro estudio, el patrón de variación temporal de la densidad bentónica y derivante fue similar y el coeficiente de Jaccard mostró un alto porcentaje de similitud taxonómica entre bentos y deriva de Chironomidae. Waters (1965) propuso que la deriva es un fenómeno denso-dependiente que vehiculiza el exceso de producción del bentos, por lo cual la estructura de las comunidades bentónicas y derivantes serán semejantes. Otras causas pueden estar interviniendo en el reclutamiento de organismos en la deriva particularmente los atributos funcionales de cada especie, tales como la morfología, el comportamiento y las preferencias de microhábitat, que influyen en la entrada a la columna de agua y en el transporte río abajo (RADER, 1997).

La proporción de las subfamilias varió entre los hábitats y estaciones del año. En bentos y deriva, Orthocladiinae presentó la abundancia más baja en invierno y en otoño la más elevada, mientras que Chironominae mostró un patrón inverso. Los taxa de Orthocladiinae se caracterizan por estar adaptados a temperaturas bajas y sus adultos pueden emerger a menores temperaturas, lo que estaría explicando la diferencia entre las altas densidades en otoño y la disminución de las mismas en invierno. Por otra parte, fueron más abundantes en rabión ya que prefieren mayor velocidad de corriente y un sustrato con abundancia de algas (A. C. Paggi, com. pers.). Las mayores abundancias de Chironominae se encontraron en corredera ya que éstos son más exitosos en aguas con poca velocidad de corriente y en sustratos con abundante acumulación de detritos, que utilizan para alimentarse (A. C. Paggi, com. pers.) y para la construcción de los tubos en que habitan (Pinder \& Reiss, 1983). Aunque son de vida libre, fijan sus tubos al sustrato, esto estaría en relación con su menor predisposición a ser arrastradas por la corriente y por lo tanto sus bajas densidades de deriva.

Los tramos próximos a las cabeceras, como el tramo estudiado en el arroyo Achiras, poseen escaso impacto humano, razón por la cual pueden constituir puntos importantes para la obtención de datos de referencia para el análisis de sitios perturbados. En la Cuenca Achiras - del Gato se construyó recientemente una presa de mediana envergadura próxima a las cabeceras destinada a embalsar las aguas con objetivos recreacionales y para provisión de agua y regulación de crecidas. Este tipo de obra produce cambios hidráulicos que alteran las características limnológicas, los procesos abióticos, y en consecuencia la biodiversidad (AlLan \& CASTILLO, 2007). De esta forma, los datos aportados por este estudio proveen valiosa información de referencia respecto de las condiciones de la biota antes de la construcción de presa. Las condiciones de calidad de los ríos pueden evaluarse utilizando invertebrados acuáticos como indicadores, especialmente Chironomidae (Rosemberg \& Resh, 1993; Marziali et al., 2006), pero 
es necesario una mejor comprensión de las preferencias ecológicas de los taxones (McGeoch \& Chown, 1998). Por ello, la ampliación del conocimiento taxonómico, biológico y ecológico del ensamble de Chironomidae posibilitara adecuar e implementar estrategias de manejo, conservación y restauración de los ecosistemas lóticos en la región central de Argentina.

Agradecimientos. Este trabajo fue subsidiado por la Secretaría de Ciencia y Técnica de la Universidad Nacional de Río Cuarto. Agradecemos al Dr. M. Arana y a la Dra. A. Oggero, por la identificación de la flora regional; a la Dra. A. Paggi, por los valiosos aportes referidos a la ecología de Chironomidae y a la Lic. L. Cibils Martina por colaborar en la edición del mapa del área de estudio.

\section{REFERENCIAS BIBLIOGRAFICAS}

Allan, J. D. 1987. Macroinvertebrate drift in a Rocky Mountain stream. Hydrobiologia 144:261-268.

Allan, J. D. \& Castillo, M. M. 2007. Stream ecology. Structure and function of running waters. Dordrecht, Springer. 400p.

Ashe, P.; Murray, D. A. \& ReIss, F. 1987. The zoogeographical distribution of Chironomidae (Insecta: Diptera). Annales of Limnology 23(1):2760.

Barbero, M. D.; Oberto, A. M. \& Gualdoni, C. M. 2013. Spatial and temporal patterns of macroinvertebrates in drift and on substrate of a mountain stream (Cordoba, Central Argentina). Acta Limnologica Brasiliensia 25(4):375-386.

Cabido, D.; Cabido, M.; Garre, S. M.; Gorgas, J. A.; Miatello, R.; Rambaldi, S.; Ravelo, A. \& Tassile, J. L. 2003. Regiones Naturales de la Provincia de Córdoba. Serie C. Publicaciones Técnicas. Agencia Córdoba. Córdoba, Dirección de Ambiente. 102p.

Corigliano, M. C.; Gualdoni, C. M. \& Oberto, A. M. 1987. Deriva de macroinvertebrados en un tramo anastomosado de un río de llanura. Revista Universidad Nacional de Rio Cuarto 7(1):89-98.

CORTES, R. M. 1992. Seasonal pattern of benthic communities along the longitudinal axis of river systems and the influence of abiotic factors on the spatial structure of those communities. Verhandlungen des Internationalen Verein Limnologie 126:85-103.

Cranston, P. S. 1995. Biogeography. In: Armitage, P.; Cranston, P. S. \& PINDER, L. C. eds. The Chironomidae. The biology and ecology of non-biting midges. London, Chapman \& Hall, p.62-84.

CrisCi, J. V. \& López Armengol, M. F. 1983. Introducción a la teoría y práctica de la taxonomía numérica. Monografía XX. Washington D.C., OEA. $131 \mathrm{p}$.

Degiovanni, S. 2005. Análisis de problemas geoambientales vinculados a los recursos hídricos en la cuenca del arroyo Achiras-del Gato. p.181222. In: Blarasin, M.; Degiovanni, S.; Cabrera, A. \& Villegas, M. eds. Aguas superficiales y subterráneas en el sur de Córdoba: una perspectiva geoambiental. Río Cuarto, Universidad Nacional de Rio Cuarto. 346p.

Di Rienzo, J. A.; Casanoves, F.; Balzarini, M. G.; Gonzales, L.; Tablada, M. \& Robledo, C. W. 2010. Infostat, software Estadística, v. 2011. Grupo InfoStat, Facultad de Ciencias Agrarias, Universidad Nacional de Córdoba. Argentina.

Dohet, A.; Dolisy, D.; Hoffmann, L. \& Dufrêne, M. 2002. Identification of bioindicator species among Ephemeroptera, Plecoptera and Trichoptera in a survey of streams belonging to the rhithral classification in the Grand Duchy of Luxembourg. Verhandlungen des Internationalen Verein Limnologie 28:381-386.

DufrêNe, M. \& LegENDRE, P. 1997. Species Assemblages and Indicator Species: The Need for a Flexible Asymmetrical Approach. Ecological Monographs 67(3):345-366.

ElLiotT, J. M. 1970. Methods of sampling invertebrate drift in running water. Annals de Limnologie 6:133-159.

EPLER, J. H. 2001. Identification manual for the larval Chironomidae (Diptera) of North and South Carolina. North Carolina, North Carolina Department of Environmental and Natural Resources, Division of Water Quality. 526p.
FLECKER, A. S. 1992. Fish predation and the evolution of invertebrate drift periodicity: evidence from Neotropical streams. Ecology 73:438-448.

GHetTI, P. F. 1986. I macroinvertebrati nell'analisi di qualitá dei corsi dáqua. Manuale di applicazione. Trento, Provincia Autonoma di Trento. $111 \mathrm{p}$

Gualdoni, C. M. \& Corigliano, M. C. 1999. Deriva de insectos y su relación estructural con el bentos. IDESIA 17:57-71.

Gualdoni, C. M. \& Oberto, A. M. 2012. Estructura de la comunidad de macroinvertebrados del arroyo Achiras (Córdoba, Argentina): análisis previo a la construcción de una presa. Iheringia, Série Zoologia 102(2):177-186.

Gualdoni, C. M.; Boccolini, M. F.; Oberto, A. M.; Principe, R. E.; Raffaini, G. B. \& Corigliano, M. C. 2009. Potential habitats versus functional habitats in a lowland braided river (Córdoba, Argentina). Annales de Limnologie - Internacional Journal of Limnology 45:1-10.

HawkINS, C. P. 1984. Substrate associations and longitudinal distributions in species of Ephemerellidae (Ephemeroptera: Insecta) from western Oregon. Freshwater Invertebrate Biology 3:181-188.

Hildebrand, S. G. 1974. The relation of drift to benthos density and food level in an artificial stream. Limnology and Oceanography 19:951-957.

Hill, M. O. 1979. TWINSPAN. A Fortran program for arranging multivariate data in an ordered two-way table by classification of the individuals and attributes. New York Cornell University.

Koetsier, P. \& Bryan, C. F. 1996. Is macroinvertebrate drift a densitydependence mechanism of the benthos in the lower Mississippi river? Journal of Freshwater Ecology 11(1):1-9.

LeHMKUHL, D. M. \& ANDERson, N. H. 1972. Microdistribution and density as factors affecting the downstream drift of mayflies. Ecology 53:661667.

Lencioni, V. \& Rossaro, B. 2005. Microdistribution of chironomids (Diptera: Chironomidae) in Alpine streams: an autoecological perspective. Hydrobiologia 533:61-76.

Marziali, L.; Gozzini, M.; Rossaro, B. \& Lencioni, V. 2009. Drift patterns of Chironomidae (Insecta, Diptera) in an arctic stream (Svalbard Islands): an experimental approach. Studi trentini di scienze naturali 84:87-96.

Marziali, L.; Lencioni, V. \& Rossaro, B. 2006. Chironomid species as indicators of freshwater habitat quality. Verhandlungen des Internationalen Verein Limnologie 29:1553-1555.

McCune, B. \& Mefford, M. J. 1999. Multivariate Analysis of Ecological Data. Version 4.25. Gleneden Beach, MjM Software.

McGeoch, M. A. \& Chown, S. L. 1998. Scaling up the value of bioindicators. Trends in Ecology \& Evolution 13:46-47.

Medina, A. I. \& PAGGI, A. C. 2004. Composición y abundancia de Chironomidae (Diptera) en un río serrano de zona semiárida (San Luis, Argentina). Revista Sociedad Entomológica Argentina 63(34):107-118

Medina, A. I.; Scheibler, E. E. \& Paggi, A. C. 2008. Distribución de Chironomidae (Diptera) en dos sistemas fluviales ritrónicos (Andinoserrano) de Argentina. Revista Sociedad Entomológica Argentina 67(1-2):69-79.

Menzie, C. A. 1981. Production ecology of Cricotopus sylvestris (Fabricius) (Diptera: Chironomidae) in a shallow estuarine cove. Limnology and Oceanography 26:467-481.

Milner, A. M. 1994. System recovery. In: Calow, P. \& Petts, G. E. eds. The Rivers Handbook. v.2. Oxford, Blackwell Scientific. 523p.

Molineri, C. 2008. Impact of rainbow trout on aquatic invertebrate communities in subtropical mountain streams of northwest Argentina. Ecología Austral 18:101-117.

Oggero A. J. \& Arana, M. D. 2012. Inventario de la Biodiversidad de Plantas Vasculares del sur de la zona serrana de Córdoba, Argentina. Hoehnea 39(2): 169-197.

Paggi, A. C. 1998. Chironomidae. In: Morrone, J. J. \& Coscarón, S. eds. Biodiversidad de Artrópodos Argentinos: Una perspectiva biotaxonómica. La Plata, Ediciones Sur, p.327-337.

1999. Los Chironomidae como indicadores de calidad de ambientes dulceacuícolas. Revista Sociedad Entomológica Argentina 58(12):202-207. 
2009. Capitulo 13- Diptera: Chironomidae. In: Domínguez, E. \& Fernández, H. R. eds. Macroinvertebrados bentónicos sudamericanos. Sistemática y biología. Tucumán, Fundación Miguel Lillo. 656p.

Pinder, L. C. V. \& Reiss, F. 1983. 10 - The larvae of Chironominae (Diptera: Chironomidae) of the Holarctic region- Keys and diagnoses. In: Wiederholm, T. ed. Chironomidae of the Holarctic region. Keys and diagnoses. Part 1: Larvae. Entomologica Scandinavica. Supplement $\mathrm{N}^{\circ} 19$. p.293-435.

Poff, N. L. \& Zimmerman, J. K. H. 2010. Ecological responses to altered flow regimes: a literature review to inform environmental flows science and management. Freshwater Biology 55:194-220.

Poi De Neiff A. \& Neiff, J. J. 2006. Riqueza de especies y similaridad de los invertebrados que viven en plantas flotantes de la planicie de inundación del río Paraná (Argentina). Interciencia 31:220-225.

Principe, R. E. \& Corigliano, M. C. 2006. Benthic, drifting and marginal macroinvertebrate assemblages in a lowland river: temporal and spatial variations and size structure. Hydrobiologia 553:303-317.

Principe, R. E.; Boccolini, M. F. \& Corigliano, M. C. 2008. Structure and Spatial-Temporal Dynamics of Chironomidae Fauna (Diptera) in Upland and Lowland Fluvial Habitats of the Chocancharava River Basin (Argentina). International Review of Hydrobiology 93(3)342-357.

Principe, R. E.; Raffaini, G. B.; Gualdoni, C. M.; Oberto, A. M. \& Corigliano, M. C. 2007. Do hydraulic units define macroinvertebrate assemblages in mountain streams of central Argentina? Limnologica 37:323-336.

RADER, R. B. 1997. A functional classification of the drift: traits that influence invertebrate availability to salmonids. Canadian Journal of Fisheries and Aquatic Sciences 54:1211-1234.
Rosemberg, D. M. \& Resh, V. H. eds. 1993. Freshwater biomonitoring and benthic macroinvertebrates. New York, Chapman \& Hall. 488p.

Ruiz-Moreno, J. L.; Ospina-Torres, R. \& Riss, W. 2000. Guía para la identificación genérica de larvas de quironómidos (Diptera: Chironomidae) de la Sabana de Bogotá. II Subfamilia Chironominae. Caldasia 22(1)15-33.

Sanseverino, A. \& Nessimian, J. L. 1998. Habitat preferences of Chironomidae larvae in an upland stream of Atlantic Forest, Rio de Janeiro State, Brazil. Verhandlungen des Internationalen Verein Limnologie 26:2141-4144.

Tejerina, E. \& Molineri, C. 2007. Comunidades de Chironomidae (Diptera) en arroyos de montaña del NOA: comparación entre Yungas y Monte. Revista de la Sociedad Entomológica Argentina 66(34):169-177.

Trivinho-Strixino, S. 2011. Larvas de Chironomidae. Guia de identificação. São Carlos, Depto Hidrobiologia/Lab. Entomologia Aquática/UFSCar. 71p.

Trivinho-Strixino, S. \& Strixino, G. 1989. Observações sobre a biologia da reprodução de um quironomídeo da região neotropical (Diptera, Chironomidae). Revista Brasileira de Entomologia 33:207-216.

WATERS, T. F. 1965. Interpretation of invertebrate drift in stream. Ecology 46(3):327-334.

Wiederholm, T. ed. 1983. Chironomidae of the Holarctic region. Keys and diagnoses. Part 1. Larvae. Entomologica Scandinavica. Supplement $\mathrm{N}^{\circ}$ 19. p.1-457. 